\title{
Direct detection of the Josephson radiation emitted from superconducting thin-film microbridges
}

Pedersen, Niels Falsig; Sørensen, O. H.; Mygind, Jesper; Lindelof, Poul Erik; Levinsen, M. T.; Clark, T. D.

Published in:

Applied Physics Letters

Link to article, DOI:

10.1063/1.88824

Publication date:

1976

Document Version

Publisher's PDF, also known as Version of record

Link back to DTU Orbit

Citation (APA):

Pedersen, N. F., Sørensen, O. H., Mygind, J., Lindelof, P. E., Levinsen, M. T., \& Clark, T. D. (1976). Direct detection of the Josephson radiation emitted from superconducting thin-film microbridges. Applied Physics Letters, 28(9), 562-564. https://doi.org/10.1063/1.88824

\section{General rights}

Copyright and moral rights for the publications made accessible in the public portal are retained by the authors and/or other copyright owners and it is a condition of accessing publications that users recognise and abide by the legal requirements associated with these rights.

- Users may download and print one copy of any publication from the public portal for the purpose of private study or research.

- You may not further distribute the material or use it for any profit-making activity or commercial gain

- You may freely distribute the URL identifying the publication in the public portal 


\title{
Direct detection of the Josephson radiation emitted from superconducting thin-film microbridges
}

\author{
N. F. Pedersen, O. H. Soerensen, and J. Mygind \\ Physics Laboratory I. The Technical University of Denmark, DK-2800 Lyngby, Denmark \\ P. E. Lindelof, M. T. Levinsen, and T. D. Clark
}

Physics Laboratory I, H.C. Orsted Institute, University of Copenhagen, Denmark

(Received 17 November 1975)

We report direct measurements of the Josephson radiation emitted in $X$ band from a superconducting thinfilm microbridge coupled to a resonance cavity. Power is emitted if one of the harmonics of the Josephson frequency is in the bandwidth of the receiver. The maximum power emitted during our experiment was $10^{-13} \mathrm{~W}$. The Josephson radiation could easily be detected at frequencies off resonance.

PACS numbers: $74.50 . \mathrm{Tk}, 73.40 . \mathrm{Jn}, 73.60 . \mathrm{Ka}$

Superconducting thin-film microbridges behave in close analogy to the resistively shunted Josephson junction model $^{1}$ (RSJ): From a practical point of view these Josephson devices have several advantages in high-frequency applications compared to other weak-link structures, e. $g_{\text {, }}$, the point contact. The thin-film microbridges have a well-defined geometry, they are easily integrated in other thin-film circuits, and they may be stored indefinitely. Hence, it is of considerable interest to investigate these bridges at mic rowave frequencies and compare the results to those obtained for point contacts.

In this letter we report the direct detection of up to $10^{-13} \mathrm{~W}$ of Josephson radiation from superconducting thin-film microbridges at $8.5 \mathrm{GHz}$. Direct observation of emitted radiation has previously been reported at $X$-band frequencies from point contacts by Dayem and Grimes, ${ }^{2}$ and very recently by Gubankov et $a l .{ }^{3}$ from variable-thickness bridges. Our results have the same general features as those found for point contacts, ${ }^{2}$ but the maximum power observed is 3 orders of magnitude smaller.

The thin-film microbridges used in this investigation were cross-scratched in 1000-2000- $\AA$-thick indium films evaporated on $7 \times 7-\mathrm{mm}$ glass substrates. ${ }^{1}$ The bridges were typically less than $0.5 \mu \mathrm{m}$ by $0.5 \mu \mathrm{m}$. The bridge was mounted in an $X$-band rectangular cavity with the junction as part of the end wall. The cavity had two resonance frequencies - one at about $8.5 \mathrm{GHz}$, the other $9.7 \mathrm{GHz}$-and the $Q$ was about 500 . The signal entered a superheterodyne receiver via three isolators followed by a low-pass filter with a steep cutoff at $16 \mathrm{GHz}$ inserted between the cavity and the balanced mixer in order to prevent the local oscillator signal and its harmonics from reaching the junction. The local oscillator power was derived from a Gunn diode. The measured noise figure of the receiver was $7.5 \mathrm{~dB}$; the i.f. amplifier had a center frequency of $70 \mathrm{MHz}$ and a 10$\mathrm{MHz}$ bandwidth. The limiting resolution in the video mode was of order $10^{-18} \mathrm{~W}$ with a $0.3-\mathrm{sec}$ time constant. The receiver was calibrated in absolute power units by means of a bolometer and a number of calibrated attenuators.

Figure 1(a) shows an $I-V$ characteristic of one of our superconducting thin-film microbridges coupled to the resonance cavity. There is an obvious deviation from the hyperbola predicted by the RSJ model, i.e., an excess current which is suggested to be connected with relaxation effects. ${ }^{4}$ Between 15 and $20 \mu \mathrm{V}$ two hardly visible bumps exist in the $I-V$ characteristic. In the $d V / d I$ curve, Fig. 1(b), these two bumps are, however, the most prominent structures. They are cavity selfinduced steps at voltages 17.5 and $19.9 \mu \mathrm{V}(8.5$ and $9.7 \mathrm{GHz}$ ). Similar cavity steps can be resolved at $\frac{1}{2}$, $\frac{1}{3}$, and $\frac{1}{4}$ of these voltages. Also, microwave-induced subharmonic steps which do not occur in the $I-V$ characteristic of the RSJ model, were present with our sample in a microwave field. Figure 1(c) shows the output

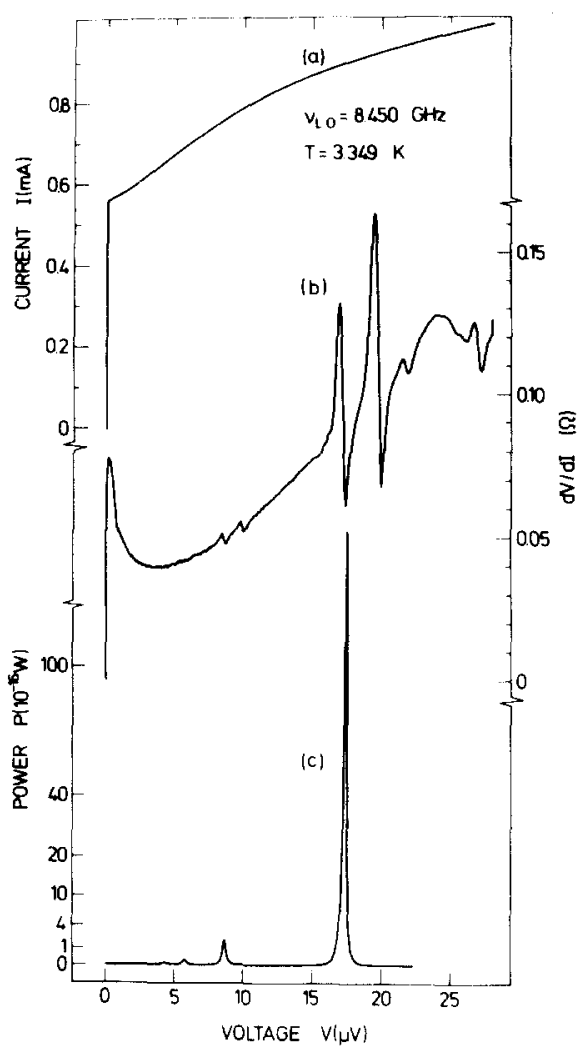

FIG. 1. (a) Current voltage $(I-V)$ characteristic, (b) first der ivative characteristic $d V / d I$, and (c) the detected microwave power output as a function of bias voltage $V$, for a superconducting thin-film indium microbridge. 


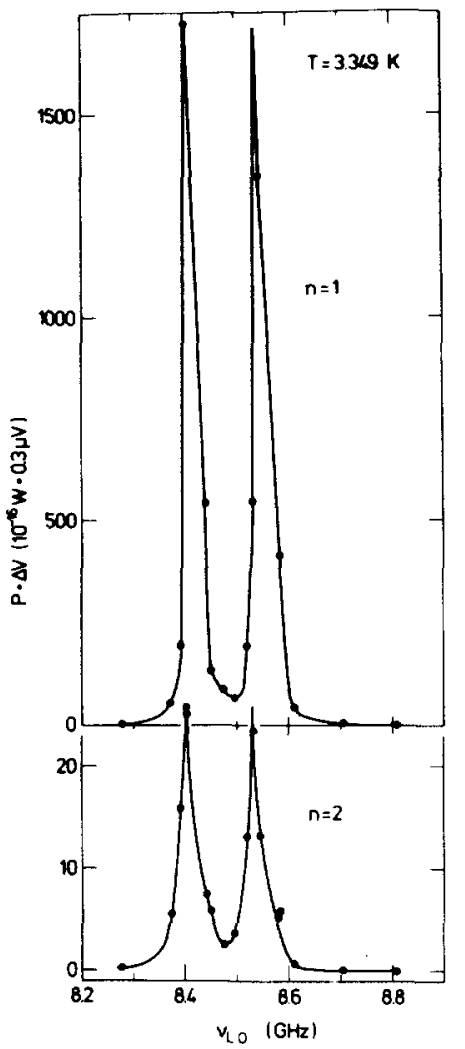

FIG. 2. "Integrated" power output for the fundamental $(n=1)$ and first subharmonic $(n=2)$ peaks depicted in Fig. 1(c) as a function of the local oscillator frequency. Note the pronounced skew of the $n=1$ peaks.

from the video detector in units of $10^{-16} \mathrm{~W}$, again as a function of the dc voltage bias. The local oscillator frequency $\nu_{\mathrm{LO}}=8.45 \mathrm{GHz}$ was chosen close to what corresponds to detection on resonance.

In Fig, 1(c) the distinct peaks in the detected signal fall at voltages $V_{n}=h \nu_{R} / 2 n e$, where $\nu_{R}$ is the receiver center frequency and $n$ is an integer. The signal centered at voltage $V_{n}$ corresponds to the $n$th harmonic of the Josephson frequency radiating into the receiver bandwidth. In the trace presented here the fourth harmonic is visible. However, at lower temperatures, as many as nine harmonics were observed. Due to current noise the linewidth of the radiation (the width at the half-power point) was typically $150-200 \mathrm{MHz}$, i.e. , comparable to the sideband separation (140 $\mathrm{MHz}$ ) of the receiver. Thus, we did not normally observe a splitting into two sidebands along the voltage axis. The maximum power which was measured at resonance was $10^{-13} \mathrm{~W}$ but in a frequency window of only $10 \mathrm{MHz}$. This emitted power is low compared to the numbers quoted for point contacts, ${ }^{2}$ but comparable to the recent observation of Gubankov et al. ${ }^{3}$ on their variable-thickness bridges.

The current noise in our experiment was essentially constant. Hence, a direct comparison of the peak heights is only meaningful if the $d V / d I$ is constant. Especially close to the cavity step this was not the case. In Figs. 2 and 3 we have, therefore, used an integrated value of the peaks in power vs voltage [Fig. 1(c)] defined as the peak maximum multiplied by the voltage (or frequency) width at half-power. The total theoretical power (without noise) at resonance can then be found by dividing by the receiver bandwidth; in this way we estimate the highest power level found to be $\sim 10^{-12} \mathrm{~W}$.

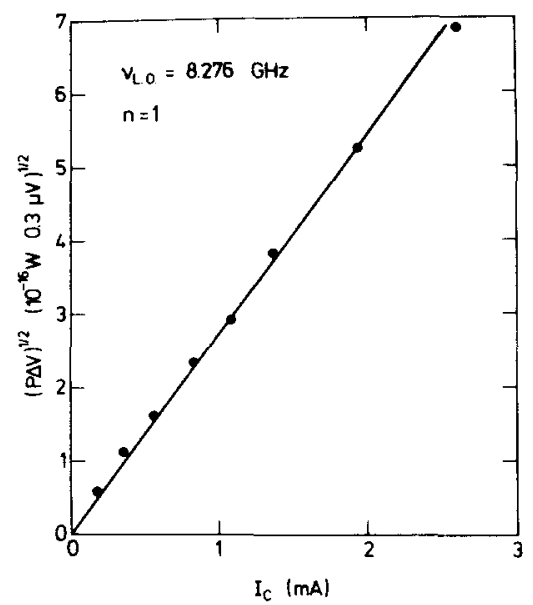

FIG. 3. Square root of the integrated power for the $n=1$ peak far from resonance as a function of the critical current of the microbridge.

In Fig. 2 the detected integral power at the fundamental Josephson frequency $(n=1)$ and second harmonic $(n=2)$ is plotted as a function of the LO frequency in the vicinity of the lower cavity resonance. The two peaks in the spectrum correspond to the two receiver sidebands $140 \mathrm{MHz}$ apart. The observed asymmetric line shapes reflects the fact that the total emitted power depends on the position of the bias point on the cavity step. ${ }^{5}$ A similar asymmetry is not resolved in the result for the second harmonic $(n=2)$ shown in Fig. 2.

The radiation was also detected at a frequency several cavity bandwidths off resonance in order to prevent small shifts of the resonance frequency from affecting the results. The emitted power was measured as a function of temperature and Fig. 3 shows the square root of integral power for the $n=1$ peak as a function of the critical current which in turn is proportional to $1-T / T_{c}$. At the highest critical current given in Fig. 3 a hysteretic jump developed in the $I-V$ characteristic and prohibited investigation of the radiation at lower temperatures. The linewidth was larger than under resonant conditions and varied from 170 to $270 \mathrm{GHz}$ as the temperature was increased from 3.141 to $3.394 \mathrm{~K}$ $\left(T_{c}=3.415 \mathrm{~K}\right)$.

According to the RSJ model the emitted power $P_{n}$ is given by

$$
P_{n}=4 R^{2} \Omega_{n}^{2}\left[\left(1+\Omega_{n}^{2}\right)^{1 / 2}-\Omega_{n}\right]^{2 n} I_{c}^{2} / n^{2} R_{0},
$$

where $R$ is the asymptotic junction resistance, $R_{0}$ is the microwave load resistance which also incorporates the coupling between junction and receiver, and $\Omega_{n}=h \nu_{R} /$ $2 n e R I_{c}$ is the normalized receiver frequency.

In the limit $\Omega_{n}^{2} \gg 1, E q$. (1) becomes

$$
P_{n}=R^{2} I_{c}^{2}\left(2 \Omega_{n}\right)^{2(1-n)} / n^{2} R_{0} \text {. }
$$

From Fig. 3 we find that $P_{1}^{1 / 2}$ is proportional to $I_{c}$ in agreement with Eq. (2) and from the slope we find that $R_{0}^{1 / 2}=7 \times 10^{-5} \Omega^{1 / 2}$, indicating a strong mismatch. If $R$ is taken as the asymptotic resistance in the experiment (Fig. 1), $R \approx 0.1 \Omega$ then, at the highest critical current $I_{c} \approx 2.6 \mathrm{~mA}$, we find $\Omega_{n}^{2} \approx 0.01$, and Eq. (2) should not be applicable in contrast to what we find. We have similar- 
ly looked at the temperature variation of the $n=2$ and $n=3$ peaks and found that the integrated power in the $n=3$ peak was proportional to $I_{c}$, whereas the $n=2$ peak had a critical current dependence intermediate between the $n=1$ and $n=3$ peaks.

The observation of the $X$-band power generation described here represent the most direct verification of the ac Josephson effect in the thin-film microbridges. The results are in close analogy with earlier observations on point contacts, ${ }^{2,5}$ but the detected power level is lower. Whether this is a general feature of thin-film microbridges (connected with the large self-inductance of the background film) is not yet clear. The recent result of Gubankov et al. ${ }^{3}$ on variable-thickness bridges may indicate this, as their power level is similar to ours. However, their results differ in having very large bandwidths for the detected radiation. Our results are in qualitative but not quantitative agreement with what is expected on basis of the resistively shunted junction model. This is not too surprising since the dc currentvoltage characteristic is also not consistent with this simple model ${ }^{4}$ It should be feasible to investigate the more subtle details of the rf properties of these bridges by direct detection at a number of frequencies.

The authors would like to thank Dr. M. Danielsen for many useful discussions on the design of the cavity. They also acknowledge B. Hermann Pettersson for the construction of the cavity and Ole Eg for sample preparation.

${ }^{1} \mathrm{P} . \mathrm{E}$. Gregers-Hansen, M. T. Levinsen, and G. Fog Pedersen, J. Low-Temp. Phys. 7, 99 (1972) ; P. E. GregersHansen and M.T. Levinsen, Phys. Rev. Lett. 27, 847 (1971).

${ }^{2}$ A.H. Dayem and C.C. Grimes, Appl. Phys, Lett, 9, 47 (1966).

${ }^{3}$ V.N. Gubankov, V.P. Koshelets, and G.A. Ovsyannikov, JETP Lett. 21, 226 (1975); Proc. LT 14, 4, 120 (1975).

${ }^{4} \mathrm{H}$. Hojgaard Jensen and P. E. Lindelof, Proc. L'T 14, 4, 152 (1975); J. Low-Temp. Phys. 23, 469 (1976).

${ }^{5} \mathrm{~A}$. Longacre, Proceedings of the Applied Superconductivity Conference, Annapolis, Md. . p. 712, 1972 (unpublished).

\title{
Nucleation of CuSi precipitate colonies in oxygen-rich silicon
}

\author{
W. K. Tice and T. Y. Tan \\ IBM System Products Division, Essex Junction, Vermont 05452 \\ (Received 19 January 1976)
}

\begin{abstract}
The nucleation of CuSi precipitate colonies in silicon containing substantial amounts of oxygen has been studied by transmission electron microscopy. It is shown that oxygen in Si can influence precipitation of $\mathrm{Cu}$ by generating prismatic dislocation loops at the interface between the Si matrix and oxygen-rich precipitates. Such loops act as nucleation sites for $\mathrm{CuSi}$ precipitates, which form star-shaped colonies upon continued solid solution decomposition.
\end{abstract}

PACS numbers: $64.80 . \mathrm{Jf}, 61.16 . \mathrm{Di}, 64.80 . \mathrm{Cz}$

It has been demonstrated by Schwuttke ${ }^{1}$ using infrared microscopy and $\mathrm{x}$-ray topography, that $\mathrm{Cu}$ precipitates preferentially in Si crystal regions of high oxygen concentration. Additional studies by Fiermans and Vennik $^{2-4}$ emphasized the part played by oxygen in providing nucleation sites for Cu precipitation. These authors, on the indirect evidence provided by macroscopic views of precipitate morphology and microprobe analysis, proposed a mechanism explaining $\mathrm{Cu}$ precipitation in $\mathrm{Si}$. This model is based on heterogenous nucleation of $\mathrm{Cu}$ precipitates on dislocations induced by indentation of the Si matrix at oxygen-containing precipitate sites. Others $s^{5-7}$ have investigated the physical and crystallographic aspects of Cu precipitates in high-purity Si. The purpose of this letter is to identify the more exact nature of $\mathrm{Cu}$ precipitate nucleation in Si containing substantial amounts of oxygen. This is accomplished through transmission electron microscopy (TEM) and diffraction analysis.

The Si material used in this work consists of $10-\Omega \mathrm{cm}$ $p$-type [001] wafers prepared from Czochralski-grown crystals. Oxygen concentration in the wafers was first

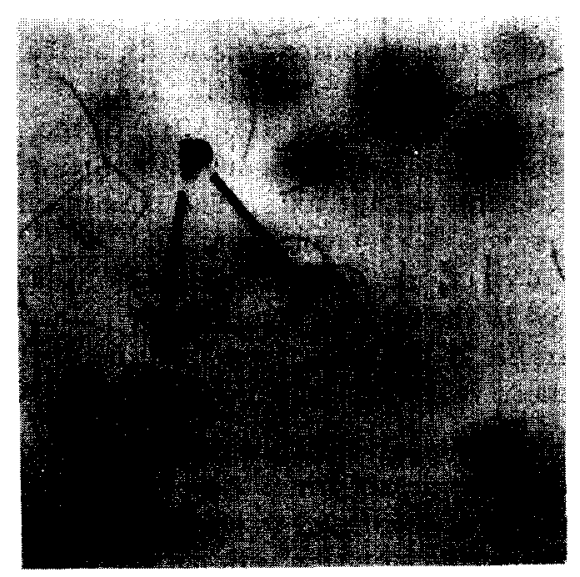

FIG. 1. TEM micrograph showing dislocation loops generated in Si by prismatic punching. Precipitates containing oxygen are designated $\mathrm{P}$. Loops here are shown as aligned sets with $\langle 110\rangle$ loop axes in four of the six possible $\langle 110\rangle$ directions. The micrograph was obtained using multiple-beam diffraction conditions. 